\title{
Modèle de la nitrification des rejets dans la Garonne au niveau de l'agglomération toulousaine
}

\author{
M. Roux - F. Simonet (*) \\ L. Masbernat - A. Line - A. Soualmia (**) \\ B. Capdeville - K. M. Nguyen $(* * *)$
}

\section{Introduction}

Les teneurs relevées en amont de Toulouse (fig. 1), aux stations de traitement d'eau potable de Clairfont et Pechdavid sont en général inférieures à $0,5 \mathrm{mg} / \mathrm{l}$. Par contre, en aval de Toulouse on relève en étiage des teneurs en $\mathrm{NH}_{4}$ de l'ordre de $3 \mathrm{mg} / \mathrm{l}$ voire plus, en particulier au droit de la prise d'eau de Lacourtensourt. Des valeurs anormales sont aussi notées à la prise d'eau de Mas-Grenier située à une cinquantaine de kilomètres à l'aval de Toulouse. Les observations indiquent donc que l'agglomération toulousaine est le centre d'importants rejets en ammoniac qui créent une situation préoccupante en période d'étiage. Deux types de rejets sont relativement bien identifiés: il s'agit d'une part des rejets de la zone industrielle sud de Toulouse, avec notamment les rejets de la Société Chimique de la Grande Paroisse (ex. : AZF (Azote et Fertilisants) dans le bras inférieur de la Garonne et d'autre part des rejets des stations d'épuration de Toulouse et de Blagnac situées en aval de l'agglomération toulousaine. Les indications des minima et des maxima en teneur en $\mathrm{NH}_{4}$ reportées sur la figure 1 illustrent bien cette situation.

Des essais préliminaires d'analyse de la cinétique de $\mathrm{NH}_{4}$ en aval de Toulouse ayant laissé craindre des effets

\begin{abstract}
d'inhibition dans l'assimilation de $\mathrm{NH}_{4}$ par Nitrosomonas, une étude systématique a été entreprise en laboratoire sur des prélèvements d'eau de Garonne. Le résultat majeur a été de montrer l'insuffisance d'une loi de cinétique de type Monod et la nécessité d'adopter un modèle de croissance de la biomasse incluant un effet d'inhibition traduisant une perte de viabilité de la biomasse active. Ce modèle de la cinétique de nitritation, défini au laboratoire, a été injecté dans un modèle de transport advectif-diffusif pour prédéterminer l'évolution de la teneur en $\mathrm{NH}_{4}$ dans la Garonne en aval de Toulouse. Le modèle de transport est couplé à un modèle de l'hydrodynamique basé sur les équations de Saint Venant (1D). On a pu ainsi tenter d'interpréter l'évolution observée dans le milieu et simuler quelques scénarios de réduction de $\mathrm{NH}_{4}$ dans la Garonne, par ensemencement des rejets.
\end{abstract}

(*) Agence de l'Eau Adour-Garonne. 84, rue du Férétra. 31400 Toulouse 61363738 .

(**) Institut de Mécanique des Fluides. Avenue du Pr C. Soula. 31400 Toulouse 61285800 .

$(* * *)$ Unité de Recherche Traitement Biologique des Eaux INSA. Avenue de Rangueil, 31077 Toulouse 61559513.

\section{Model of nitrification of waste into the Garonne river at the level of the City of Toulouse}

For several years now the amount of nitrogen in the Garonne, downstream from Toulouse, has been worrying the people managing water supplies. A nitration model is presented, with a law for the growth of the biomass including an inhibition effect, coupled to a hydrodynamic model based on the Saint-Venant equations; the kinetics is propped up with batch experiments. The complete model is validated from observations carried out on the ground. The tool thus made will allow managers to simulate different scenarios and the impact of pollution on the Garonne. 


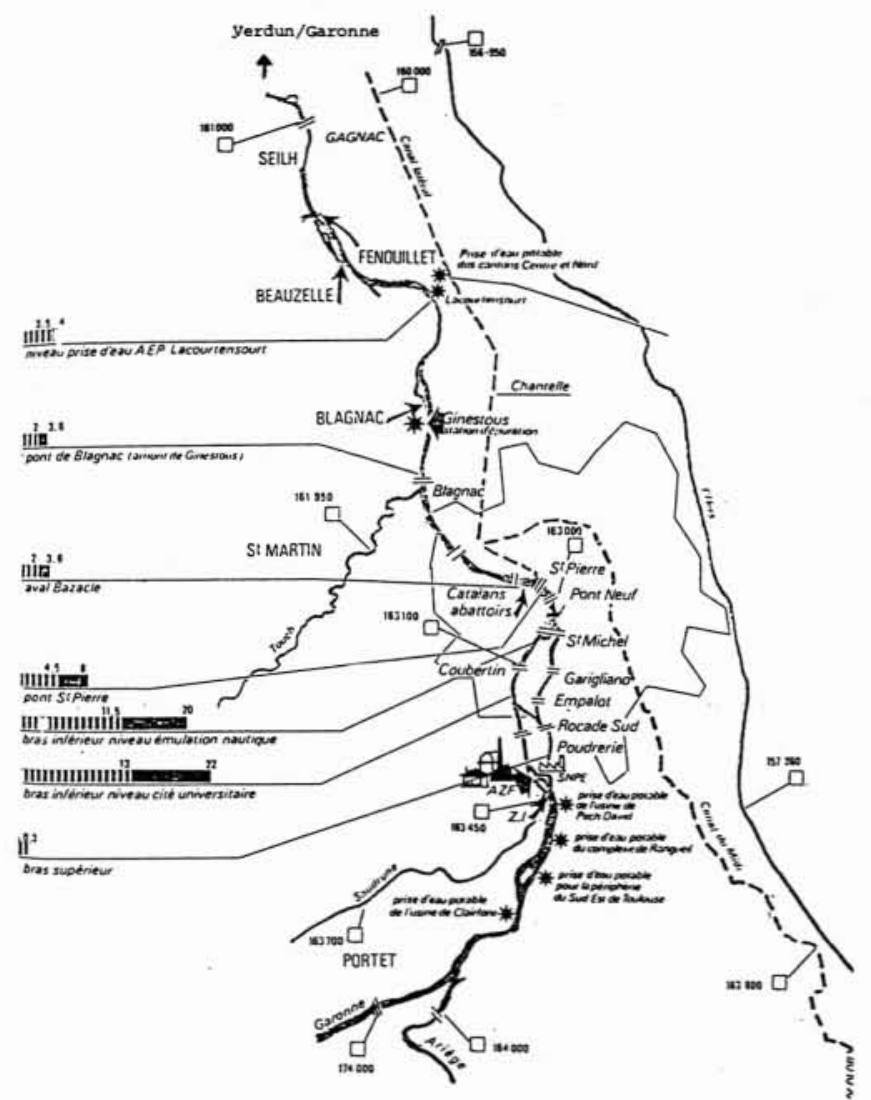

1. La Garonne au niveau de l'agglomération toulousaine.

2. Cinétique de croissance de nitrosomonas et d'assimilation de $\mathrm{NH}_{4}$

Les deux étapes de la nitrification sont bien connues: Etape de nitritation par Nitrosomonas:

$$
\mathrm{NH}_{4}^{+}+3 / 2 \mathrm{O}_{2} \rightarrow \mathrm{NO}_{2}^{-}+\mathrm{H}_{2} \mathrm{O}+2 \mathrm{H}^{+}+\mathrm{E} .
$$

Etape de nitratation par Nitrobacter :

$$
\mathrm{NO}_{2}^{-}+1 / 2 \mathrm{O}_{2} \rightarrow \mathrm{NO}_{3}^{-}+\mathrm{E} \text {. }
$$

Tableau 1. Définition des essais.

\begin{tabular}{|c|c|c|l|l|}
\hline $\mathrm{N}^{*}$ & $S_{0}=\mathrm{N}-\mathrm{NH}_{4}$ & MES Réf. & $\mu_{N} h^{\prime}$ & $X_{0} \mathrm{mg} / 111$ \\
\hline 1 & 34 & 1 & 0,02 & 0,01 \\
2 & 34 & 10 & 0,009 & 0,2 \\
3 & 34 & 15 & 0,007 & 0,5 \\
4 & 36,9 & 20 & 0,009 & 0,5 \\
5 & 37,5 & 30 & 0,005 & 1,0 \\
$1 / 1$ & 10 & 20 & 0,004 & 1,5 \\
$1 / 2$ & 15 & 20 & 0,004 & 1,3 \\
$1 / 3$ & 25 & 20 & 0,0065 & 1,0 \\
$1 / 4$ & 30 & 20 & 0,0065 & 1,0 \\
\hline
\end{tabular}

Notre étude s`attache à analyser la première étape de la modélisation de la cinétique de nitritation (1) de $\mathrm{N}-\mathrm{NH}_{4}$. Des expériences ont été réalisées à l'INSA de Toulouse sur des échantillons d'eau de Garonne avec des ensemencements variables en biomasse du groupe Nitrosomonas. Dans le tableau 1 , on a caractérisé les essais réalisés par la teneur initiale $\mathrm{S}_{0}$ en $\mathrm{N}-\mathrm{NH}_{4}$ et la teneur en MES (matières en suspension) caractéristique de l'ensemencement initial.

\subsection{Test du modèle de Monod}

Le modèle de Monod est couramment utilisé pour représenter la cinétique d'un substrat de concentration $S$ par une biomasse de concentration $X$ :

$$
\begin{aligned}
& \frac{\mathrm{d} X}{\mathrm{~d} t}=\mu_{N} \frac{S}{S+S_{L}} X \\
& \frac{\mathrm{d} S}{\mathrm{~d} t}=-\frac{1}{Y} \frac{\mathrm{d} X}{\mathrm{~d} t} .
\end{aligned}
$$

Les paramètres intervenant dans le modèle de Monod sont: $Y$, le taux de conversion du substrat en biomasse ; $S_{L}$ : concentration seuil de demi-saturation $; \mu_{N}$ : taux de croissance de la biomasse, à ajuster. On prendra :

$$
\begin{aligned}
& Y=0,05 \\
& \mathrm{mg} / 1<S_{L}<3,5 \mathrm{mg} / \mathrm{P} \\
& h^{-1}<\mu_{N}<0,1 h^{-1} .
\end{aligned}
$$

Les équations (3), (4) sont résolues numériquement avec des valeurs initiales de la teneur en substrat $S_{0}$ et en biomasse $X_{0}$. A $t=0$ :

$$
S=S_{0} ; X=X_{0} .
$$

Sur les figures 2 à 5 , on a représenté quelques résultats types d'évolution de $\mathrm{N}-\mathrm{NH}_{4}$ et de Nitrosomonas après calage de $\mu_{N}$ et $X_{0}$ pour les valeurs mesurées de $\mathrm{N}-\mathrm{NH}_{4}$. On constate qu'il est possible d'ajuster le modèle de Monod sur les résultats expérimentaux mais avec des valeurs de $\mu_{N}$ beaucoup plus faibles que celles normalement observées (7). On constate aussi que les valeurs de $X_{0}$ sont beaucoup plus faibles que les teneurs en MES initiales (tabl. I) ce qui indique qu'une faible partie de la biomasse est active.

\subsection{Modèle à effet d'inhibition ou de vieillissement associé au concept de viabilité}

Chang (1988) [1] propose une nouvelle approche de la modélisation en tenant compte des principaux constituants intervenant dans la mesure globale de la biomasse en terme de MES : il introduit un coefficient de viabilité de l'ensemencement et des effets de perte de viabilité des micro-organismes responsables de la croissance. La viabilité est le rapport du nombre de micro-organismes viables (capables de se diviser et de se reproduire) au nombre total de micro-organismes.

$X_{V}$ désignant la teneur en biomasse viable on exprime sa cinétique sous la forme:

$$
\left(\mathrm{d} X_{V} / \mathrm{d} t\right)=r_{c}-r_{I N} .
$$




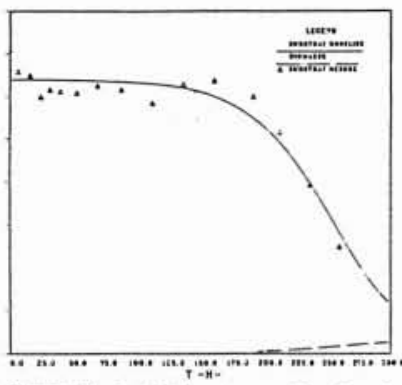

Figure 2. Evolution temporelle du substrat

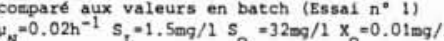

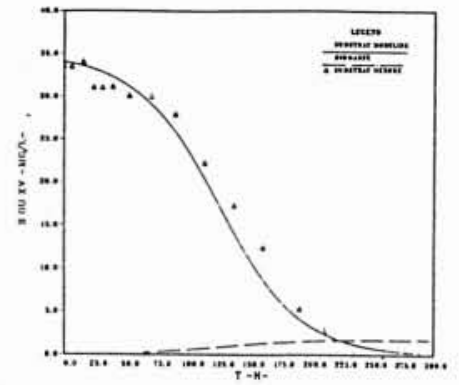

Fiqure 3. Evolution temporelle du substrat compare aux mesures en batch
(Essai $\mathrm{n}^{0}$ 2). $\mu_{\mathrm{n}}=0.05 \mathrm{~h}^{-1}, \mathrm{~s}_{\mathrm{n}}=1,5 \mathrm{mg} / 1$ $s_{0}=34 \mathrm{mg} / 1 \quad x_{0}=0.2 \mathrm{mg} / \mathrm{A}$

AZOTE DANS LA GARONYE

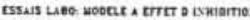

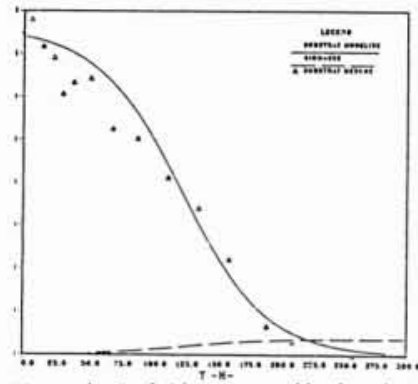

PLqure 4. Evolution temporelle du substrat

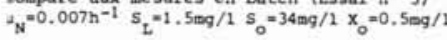

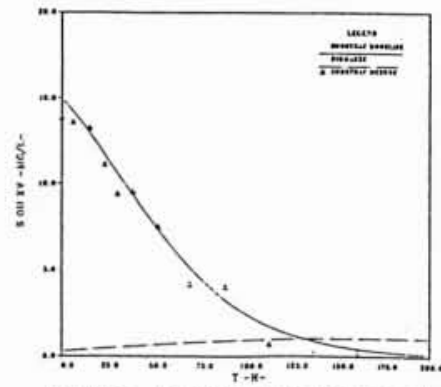

Figure 5. Evolution temporelle du subs trat comparé aux mesures en batch (fosal

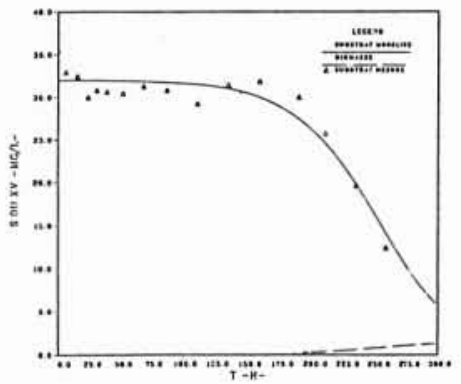

Figure 6. Evolution temporelle du substra: comparé aux mesures en batch (Essai i
$u_{\mathrm{N}}=0.03 \mathrm{~h}$

ATOTE DANS LA GARONYE

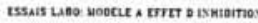

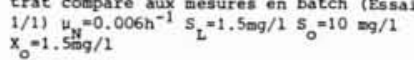

2 à 5. Evolution temporelle du substrat comparée aux valeurs en batch avec un modèle de Monod.

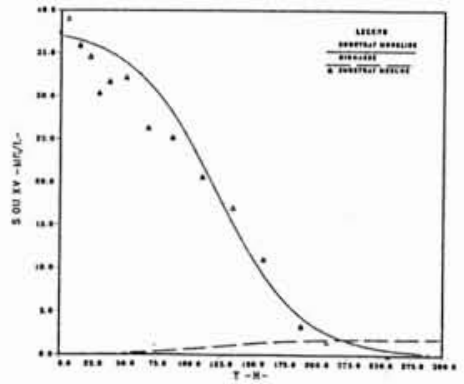

Figure $B$. Evolution temporelle du substrat comparb apx mesures en bateh (essai 3) $\psi_{N}=0.03 \mathrm{~h}^{-1} \mathrm{~s}_{\mathrm{O}}=37 \mathrm{mg} / 1 \quad \mathrm{x}_{0}=0.045 \mathrm{mg} / 1$

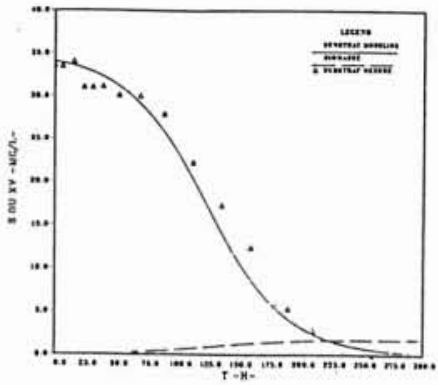

Figure 7. Evolution temporelle du substrat comparé aux mesures en batch (Essal 2) ${ }_{\mathrm{N}}=0.03 \mathrm{~h}^{-1} \mathrm{~s}_{\mathrm{O}}=34 \mathrm{mg} / 1 \quad \mathrm{x}_{\mathrm{O}}=0.045 \mathrm{mg} / 1$

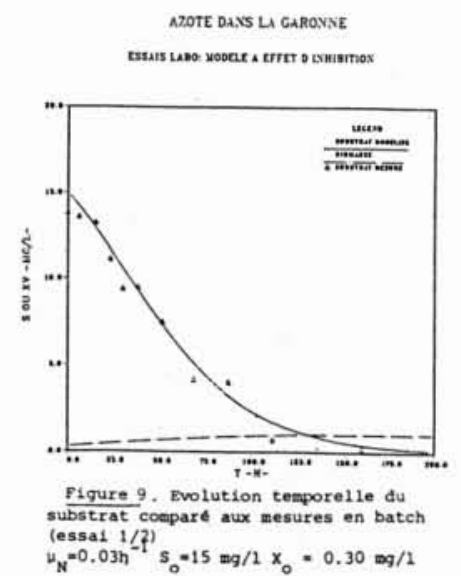

6 à 9. Evolution temporelle du substrat comparée aux valeurs en batch avec un nouveau modèle cinétique.

On définit pour le taux de croissance $r_{c}$ des bactéries viables une cinétique d'ordre 0 par rapport au substrat et d'ordre 1 par rapport à la biomasse (loi classique de multiplicité cellulaire) :

$$
r_{c}=\mu_{N} X_{V} .
$$

Par contre la cinétique de perte de viabilité est d'ordre 2 ; elle dépend de la concentration cellulaire, de l'accumulation d'inhibiteurs de concentration $I$ :

$$
r_{I N}=\gamma I X_{V} .
$$

Et en admettant que la concentration en inhibiteurs est proportionnelle à la concentration cellulaire on aboutit au modèle d'évolution :

$$
\frac{\mathrm{d} X_{V}}{\mathrm{~d} t}=\mu_{N} X_{V}-C X_{V}^{2} .
$$

A l'équilibre $\frac{\mathrm{d} X_{V}}{\mathrm{~d} t}=0, X_{V}=X_{V \text { Max }}$, d'où

$$
C=\frac{\mu_{N}}{X_{\mathrm{Max}}} .
$$

En batch $X_{V}$ est connue par intégration de (4) :

$$
\begin{aligned}
& \frac{\mathrm{d} X_{V}}{\mathrm{~d} t}=-Y \frac{\mathrm{d} S}{\mathrm{~d} t}, \text { d'où } \quad X_{V}-X_{V 0}=-Y\left(S_{0}-S\right) \\
& \text { soit : } \quad X_{V \operatorname{Max}}=Y S_{0}+X_{V 0}=X_{V}+Y S .
\end{aligned}
$$

Dans le milieu naturel, hors de léquilibre la valeur de $X_{V \text { Max }}$ à associer à chaque échantillon dans chaque section est :

$$
X_{V \operatorname{Max}}=X_{V}+Y S .
$$

Tenant compte de (12), (13), (15) le modèle proposé pour la cinétique de Nitrosomonas viable et de l'assimilation de $\mathrm{N}-\mathrm{NH}_{4}$ est le suivant:

$$
\begin{aligned}
\frac{\mathrm{d} X_{V}}{\mathrm{~d} t} & =\mu_{N} X_{V}-\mu_{N} \frac{X_{V}}{X_{V}+Y S} X_{V} \\
\frac{\mathrm{d} S}{\mathrm{~d} t} & =-\frac{1}{Y} \frac{\mathrm{d} X_{V}}{\mathrm{~d} t} .
\end{aligned}
$$

Ce modèle a été utilisé pour traiter les essais de laboratoire. On constate figures 6 à 9 qu'une interprétation satisfaisante des expériences est possible avec une valeur constante du taux de croissance : $\mu_{N}=0,03 h^{-1}$.

C'est le modèle (16), (17) à l'effet d'inhibition qui a été retenu pour représenter l'évolution de l'azote ammoniacal dans la Garonne. Notons que l'équation (16) s'écrit aussi :

$$
\frac{\mathrm{d} X_{V}}{\mathrm{~d} t}=\mu_{N} \frac{Y S}{X_{V}+Y S} X_{V} .
$$

Le modèle (18) est à rapprocher du modèle de Monod avec une "constante seuil" $S_{L}$ variable: $S_{L}=X_{V} / Y$. 


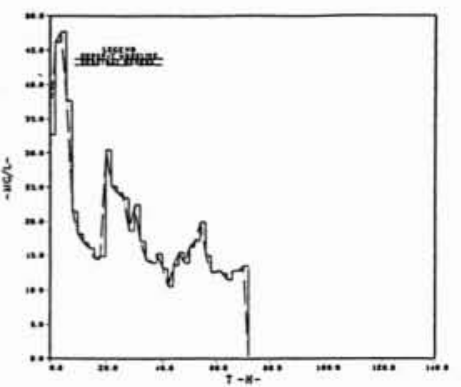

Piqure 10. Evolution de 1a teneur en

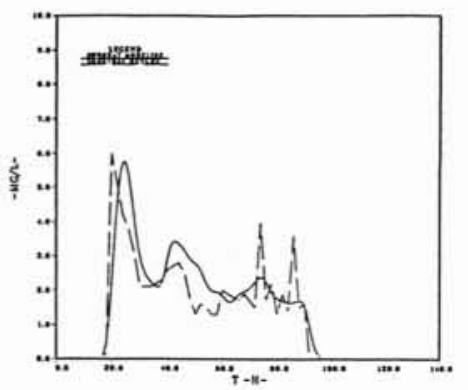

Yigure il comparaison des évolutions de 1a teneur en azote mesurees et simulfes avec un modele conservatif
$\mathrm{x}_{\mathrm{y}}=\mathrm{O}$ a $\mathrm{AZF}$ et a Ginestous

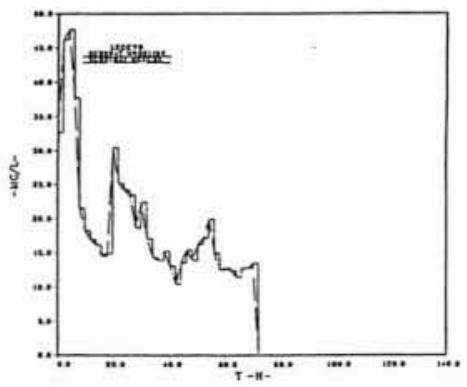

Figure 14. Evolution de la teneur en azote mesurfe dans le bras inférieur

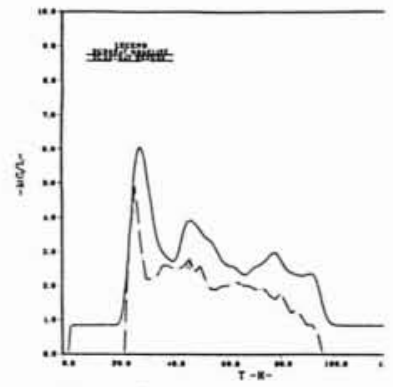

Fiqqure 15. Comparaison des évolut: 1a teneur en azote sesurées et simule $\mathrm{x}_{\mathrm{v}}=10 \mathrm{mg} / 1 \mathrm{a}$ Ginestous
AZOTE DANS LA GARONNE LACOURTENSOUKT

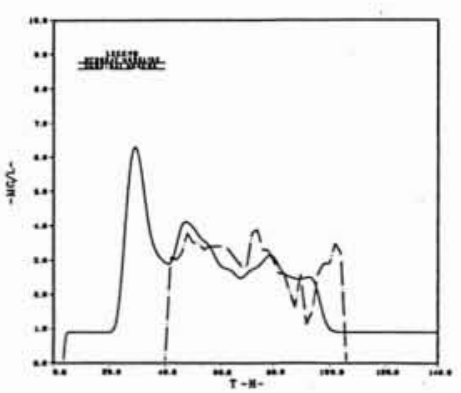

Fiqure 12 Comparal son des tvolutions de ia
teneur efn azote mesurées et simulées avec un

modele conservatif
$\mathrm{x}_{\mathrm{v}}=0$ a ALF et a Ginestous
AZOTE DANS LA GARONNE: VERDUN

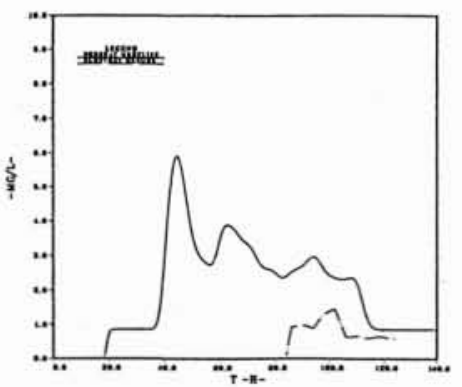

Figure 13. Comparalson des Evolutions de avec un modele conservatif
10 à 13. Comparaison des évolutions de la teneur en azote avec un modèle conservatif.
AZOTE DANS LA GARONNE: LACOURTENSOURT

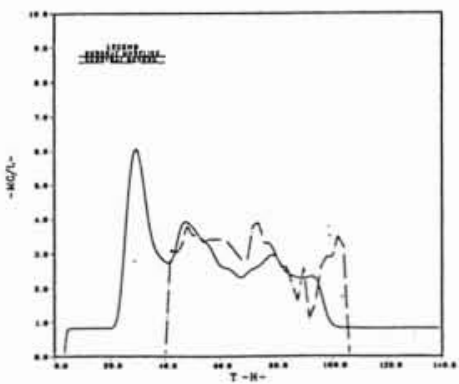

Fiqure 16. Comparaison des évolutions de 1a teneur en azote mesurfes et simuletes avec un modele cinetique sans ensemencement a AZF, $x_{v}=10 \mathrm{mg} / 1$ a Ginestous

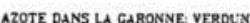

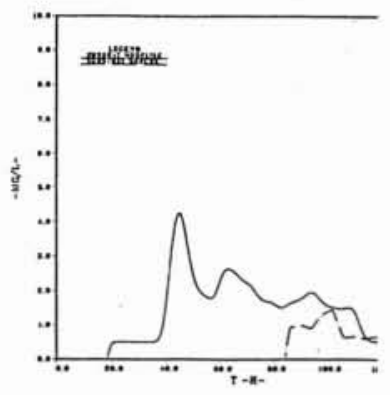

Fiquie 17. Comparaison des fvolutic 1a teneur en azote mesurees et sim avec un modele cinetique sans ense a AZF, $x_{y}=10 \mathrm{gg} / 1$ a Ginestous

14 à 17. Comparaison des évolutions de la teneur en azotc avec un modèle cinétique sans ensemencement à $A Z F$.

\section{Modèle (1D) de l'azote ammoniacal dans la Garonne en aval de Toulouse}

Les équations de base de la modélisation de l'évolution de $\mathrm{N}-\mathrm{NH}_{4}$ en rivière, sont les équations de transport advectif-diffusif de quantité de mouvement et de masse, les différentes grandeurs étant intégrées dans la section. Le modèle de l'hydrodynamique s'écrit :

$$
\begin{gathered}
\frac{\partial A}{\partial t}+\frac{\partial A U}{\partial X}=-\frac{M}{\rho} \\
\frac{\partial A U}{\partial t}+\frac{\partial A U^{2}}{\partial X}=-g \frac{\partial J}{\partial X}+P_{F} \frac{\tau_{F}}{\rho} \\
\frac{\tau_{F}}{\rho}=\frac{1}{2} C_{F} U^{2} 2 C_{F}=\frac{1}{\left(2 \log \frac{R_{H}}{R_{S}}+1,74\right)^{2}}
\end{gathered}
$$

représente la section hydraulique $A(H)$, le périmètre $P_{F}(H)$, le rayon hydraulique $R_{H}(H)$ sont calculés par tronçons à partir d'une section trapézoïdale équivalente déduite des relevés topographiques du fond. Le modèle de la teneur $S$ en $\mathrm{N}-\mathrm{NH}_{4}$ et de la tenseur $X_{V}$ en Nitrosomonas viable s"écrit :

$$
\begin{aligned}
\frac{\partial A X_{V}}{\partial t}+\frac{\partial A U X_{V}}{\partial X}=\frac{\partial}{\partial x}(D & \left.\frac{\partial X_{V}}{\partial X}\right)+ \\
& +\mu_{N} \frac{Y_{S}}{X_{V}+Y S} X_{V}+S_{X} \\
\frac{\partial A S}{\partial t}+\frac{\partial A U S}{\partial X}=\frac{\partial}{\partial x}\left(D \frac{\partial S}{\partial X}\right)- & -\mu_{N} \frac{S}{X_{V}+Y S} X_{V}+S_{S} .
\end{aligned}
$$

$S_{X}$ et $S_{S}$ représentent des sources ou puits traduisant des échanges continus avec le fond ou le bassin versant. Les équations (19) à (20) sont résolues numériquement en utilisant le code de calcul "Geraldine " mis au point à I'IMFT [2]. Ce code est utilisé pour simuler l'hydrodynamique de la Garonne, de Muret à Verdun-sur-Garonne. La rugosité a été calée à partir de trois hydrogrammes $Q(H)$ déterminés par tarage dans trois sections à Portetsur-Garonne, au Pont de l'Embouchure, à Verdun-surGaronne. Les valeurs de la rugosité équivalente $k_{s}$ sont respectivement de $0,8,0,2,0,06 \mathrm{~m}$; cette variation traduit une évolution visible du lit de la Garonne avant la confluence avec l'Ariège jusqu'à sa confluence avec le Tarn. Tous les détails sur le calage du modèle hydrodynamique sont donnés dans [3]. 


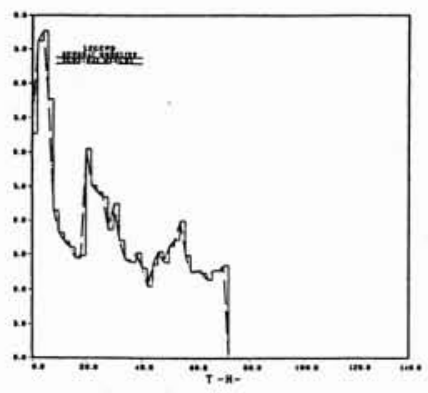

Figure 18. Evolution de la teneur en azote mesurée dans le bras inférieur

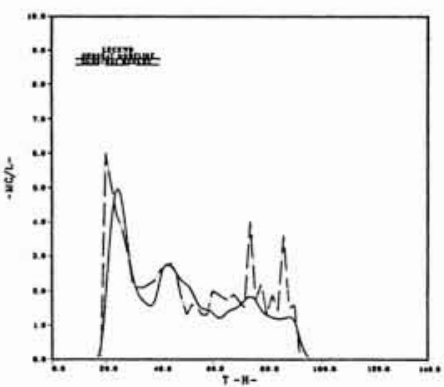

Fiqure 19. Comparaison des fvolutions de is teneur en azote mesurées et simulées avec un modèle cinétique, avec ensemence$x_{v}=10 \mathrm{~m} / 1 \mathrm{x} v=0.5 \mathrm{mg} / 1$ et a Ginestou

AZOTE DANS LA GARONNE: VERDUN

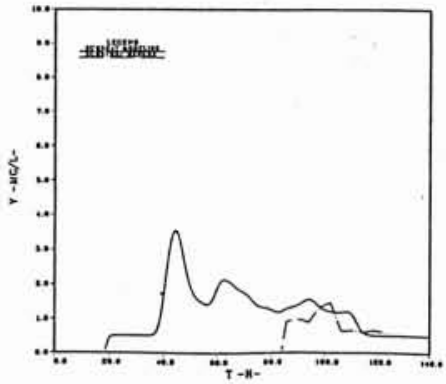

Figure 21. Comparaison des evolutions de la teneur en azote mesurées et simulées ment a AZF : $x_{y}=0.5 \mathrm{mg} / 1$ et a Ginestous $\mathrm{x}_{\mathrm{y}}=10 \mathrm{mg} / 1$
Figure 20 . Comparaison des évolutions avec un modale cinétique, avec enstences a AZF : $\mathrm{x}_{\mathrm{y}}=0,5 \mathrm{mg} / 1$ et a Ginestous $x_{v}=10 \mathrm{mg} / 1$
18 à 21. Comparaison des asolutions de la tenter en azote avec un modèle cinétique avec ensemencement à $A Z F$.
Trois simulations ont été réalisées avec un coefficient de croissance de la biomasse viable constant $\mu_{N}=$ $0,03 h^{-1}$ et $Y=0,05$. Les seuls paramètres d'ajustement sont alors les teneurs initiales en biomasse viable dans les rejets d'AZF et de Ginestous (on néglige les échanges avec le fond et le bassin versant $S_{X}=0, S_{S}=0$ ).

$1^{\text {re }}$ simulation (fig. 10 à 13 )

$X_{V}=0$ à AZF. $X_{V}=0$ dans le rejet de Ginestous.

Dans ce cas l'azote ammoniacal est conservatif: les résultats présentés sur les figures 10 à 13 indiquent que la reconstitution de l'hydrodynamique semble correcte, qu'à partir de Lacourtensourt les observations ont commencé trop tard et on "n'a pas vu " la pointe de pollution et que la dégradation de $\mathrm{NH}_{4}$ est surtout sensible à Verdun-sur-Garonne.

$2^{\mathrm{e}}$ simulation (fig. 14 à 17)

$X_{V}=0$ à AZF. $X_{V}=10 \mathrm{mg} / \mathrm{l}$ dans le rejet de Ginestous.

On constate que l'ensemencement du rejet de Ginestous est détectable à partir de Lacourtensourt et nettement visible à Verdun.

$3^{\mathrm{c}}$ simulation (fig. 18 aे 21)

$X_{V}=0,5 \mathrm{mg} / 1$ à AZF. $X_{V}=10 \mathrm{mg} / 1$ dans le rejet de Ginestous.

La nitritation de $\mathrm{N}-\mathrm{NH}_{4}$ est sensible dans le bras inférieur en comparant les figures 11 et 19 à l'Embouchure. L'effet de l'ensemencement à AZF, s'il explique bien les teneurs observées à Verdun, figure 21 , paraît surestimer le niveau de dégradation à Lacourtensourt.

\section{Résultats de simulation}

On a choisi de tester le modèle de l'azote ammoniacal sur un ensemble d'observations réalisées par l'Agence de Bassin Adour Garonne en septembre 1987. Ces mesures révèlent $\mathrm{N}-\mathrm{NH}_{4}$ supérieures à $45 \mathrm{mg} / \mathrm{l}$ dans le bras inférieur ; un suivi de cette pollution a été réalisé dans plusieurs sections en aval jusqu'à Verdun-sur-Garonne. Le modèle (22), (23) a été utilisé dans les conditions suivantes :

- Hydrodynamique: régime permanent - débit de $42 \mathrm{~m}^{3} \mathrm{~s}^{-1}$ à l'entrée du bras supérieur et $8 \mathrm{~m}^{3} \mathrm{~s}^{-1}$ dans le bras inférieur.

- Azote Ammoniacal : on a introduit deux rejets d'azote ammoniacal à $\mathrm{AZF}$ et à la station de Ginestous.

- Rejet AZF : il est défini par la teneur en azote dans le bras inférieur mesuré lors du passage accidentel de septembre 1987. On disposait d'un relevé continu pendant 70 heures qui est utilisé comme donnée d'entrée dans le bras inférieur pour les trois simulations présentées ici (fig. 10, 14, 18).

- Rejet Ginestous : il est maintenu constant avec un débit de $1,5 \mathrm{~m}^{3} / \mathrm{s}$ et une teneur en $\mathrm{N}-\mathrm{NH}_{4}$ de $30 \mathrm{mg} / \mathrm{l}$.

\section{Conclusion}

Sans prétendre avoir levé toutes les difficultés liées à la compréhension des phénomènes de nitrification dans la Garonne, on peut estimer qu'une avancée sensible a été réalisée dans cette voie. Il semble d'abord que le modèle de nitrification proposé, après les essais de laboratoire et son utilisation dans le milieu, paraisse plus réaliste que le modèle de Monod. Malgré le caractère incomplet des observations in situ, les essais de détermination des biomasses Nitrosomonas initiales au droit des principaux rejets paraissent cohérents vis-à-vis des essais de laboratoire.

L'utilisation du modèle permet aussi une critique de l'échantillonnage spatiotemporel sur le terrain et les prochaines observations devraient être conduites de manière plus rationnelle. D'ores et déjà cette étude met bien en évidence les effets qu'un prétraitement des effluents pourraient avoir complémentairement à la capacité de nitrification du milieu. 


\section{Références}

CHANG J. (1988). - Etude cinétique et modélisation de la croissance d'une population mixte sur substrat complexe par couplage d'un réacteur discontinu à un spectromètre de masse. Thèse Doctorat INSAT n* 71, 1-180.

Liné A., Masbernat L., Prodhomme M. T. (1988). - Modélisation mathématique de l'hydrodynamique et du transport de constituants dans les systèmes aquatiques à surface libre. Application à la Garonne, de Muret à la confluence avec le Tarn. Rapport de fin de contrat n* 372.

Ajdor Y., Dalmayrac S., Fabre J. (1987), - Dispersion d'un soluté passif. Fermeture des flux par une équation de transport. Journal de Mécanique théorique et appliquée. Vol. 6, n" 2, pp. 209-210.

CAZARD I. (1987). - Transport de polluants dans la Garonne. Rapport interne. EME 254, IMFT.

FABRE J. - Calcul des courants et tirants d'eau dans un réseau de chenaux à sections variables, en fonction du temps. Rapport interne EME-IMFT. 